LPTHE 92-42

\title{
Regularization and Renormalization of Chern-Simons Theory*
}

\author{
G. Giavarini, ${ }^{\text {a) }}$ C. P. Martin ${ }^{\text {b) }}$ and F. Ruiz Ruiz ${ }^{\mathrm{c}}$ \\ a) L.P.T.H.E., Université Paris VI-VII, 2 Place Jussieu, 75251 Paris Cedex 05, France. \\ b) Department of Mathematics and Statistics, University of Guelph, Guelph, Ontario N1G 2W1, Canada. \\ c) Niels Bohr Institute, University of Copenhagen, Blegdamsvej 17, DK-2100 Copenhagen Ø, Denmark.
}

The topological field theory most familiar both to physicists and to mathematicians is surely Chern-Simons theory. The classical Chern-Simons euclidean action, for a principal $G$-bundle $P$ over an oriented three manifold $M$, is given by

$$
S_{\mathrm{CS}}[A]=-\frac{i k}{4 \pi} \int_{M} \operatorname{Tr}\left(A \wedge \mathrm{d} A+\frac{2}{3} A \wedge A \wedge A\right)
$$

where $A$ is the gauge connection on $P \rightarrow M$ and $\operatorname{Tr}$ represents a $G$-invariant bilinear form on $\mathfrak{g}$, the Lie algebra of $G$. In the following we shall assume $G=S U(N)$ and $A$ taking values in the fundamental representation of $\mathfrak{s u}(N)$, with the (antihermitian) generators $T^{a}$ normalized so that $\operatorname{Tr}\left(T^{a} T^{b}\right)=\frac{1}{2} \delta^{a b}$. The field theory with action (1) is "topological" in the sense that it does not depend on the metric chosen on $M$ and general covariance is thus manifest.

The action (1) is invariant under the infinitesimal gauge transformation $A \rightarrow A+D \eta$, where $\eta$ is a $\mathfrak{s u}(N)$ valued function on $M$ and $D=d+A$ is the covariant derivative with respect to $A$. The field theory described by $S_{\mathrm{CS}}[A]$ is then particularly appealing since it provides an alternative to the usual Yang-Mills action for describing gauge fields in three dimensions. It is by now a well established fact that matter fields coupled to a Chern-Simons gauge field give rise to particles with fractional statistics (the so called anyons) that might be relevant for the description of phenomena such as the fractional quantum Hall effect or high $T_{c}$ superconductivity [1]. In the following however, our main concern will be the pure gauge theory with action given by (1).

As usual in field theory, one is interested in the computation of the vacuum expectation value of observables. It is clear that gauge invariant local operators are not necessarily generally covariant and consequently they are not appropriate for the case at hand. One must then look for non-local, metric independent and gauge invariant objects. Physicists

^ Talk presented by G. Giavarini at the NATO AWR on "Low dimensional Topology and Quantum Field Theory", 6-13 September 1992, Cambridge (UK). 
working on more standard gauge theories (like the old good QCD in 4 dimensions) have been for a long time dealing with the computation of observables having the above properties. They called these observables "Wilson loops", but mathematicians seem to prefer the name of "holonomies" of the connection $A$. Given a closed curve $C$ in $M$, a Wilson loop is defined as

$$
\mathcal{W}(C)=\operatorname{Tr} P \exp \oint_{C} A
$$

The expectation value of a collection $\left\{C_{i}\right\}$ of Wilson loops is given by the Feynman path integral

$$
Z\left(M ; C_{i}\right)=\int \mathcal{D} A \prod_{i} \mathcal{W}\left(C_{i}\right) \mathrm{e}^{-S_{\mathrm{CS}}[A]}
$$

In the simple case in which no Wilson loop appears in (2) one gets the partition function $Z(M)$ of the theory.

Owing to the intrinsecally topological character of $S_{\mathrm{CS}}[A]$ one expects the functors $Z\left(M ; C_{i}\right)$ to give back topological invariants of $M$ and $\left\{C_{i}\right\}$. To make sure this is indeed the case we might content ourselves with the study of the formal properties of the path integral (2). However, if some explicit result has to be obtained, one cannot avoid to give concrete meaning to eq.(2). This in practice signifies that we must quantize Chern-Simons theory (CST). Taking $M=S^{3}$, it was shown in ref. [2], by using input results coming from conformal quantum field theory, that the expectation values of Wilson loops satisfy the same skein relation as the Jones polynomials [3] and can be identified with the latter. More precisely, $Z\left(S^{3} ; C\right) / Z\left(S^{3}\right)$ with the standard framing is the Jones polynomial for $C$ in the variable $q=\exp \left\{2 \pi i /\left(k+\operatorname{sign}(k) c_{V}\right)\right\}$, where $k$ is the classical parameter appearing in (1) and $c_{V}$ is the second Casimir operator in the adjoint representation of $S U(N)$. With our normalization choice for the group generators, $c_{V}$ is simply $N$. In this way CST provides a three dimensional framework for computing knot invariants. The partition function itself is a topological invariant of $M$, as explicitly checked in ref. [4].

The fact that the observables of CST are functions of the combination $k+c_{V}$, rather than $k$, has a clear quantum mechanical interpretation. It relies on the precise correspondence between CST on $M$ and the Wess-Zumino-Witten model on $\partial M$, as first displayed in [2]. Indeed, $k$ can be identified with the level of the affine $S U(N)$ current algebra on $\partial M$. Then the Sugawara form of the energy-momentum tensor implies the shift

$$
k \rightarrow k+\operatorname{sign}(k) c_{V}
$$

We shall discuss in a moment the reasons leading to quantization conditions for the parameter $k$ in CST which, just like the central charge in the representation theory of affine algebras, must take integer values. 
One may think of getting a clue to the understanding of the shift (3) without resorting to the aid of conformal field theory. In a semiclassical quantization of CST, eq.(3) can be recovered as the radiative correction to the parameter $k$ at order $\hbar$ [2]. From this simple remark, it should appear clear the relevance of perturbation theory to acheive a complete understanding of the quantum theory defined by (1). Perturbation theory, moreover, provides a very direct tool for obtaining intrinsically three dimensional integral representations of knot and link invariants that generalize Gauss' formula for the linking number of two closed curves, as illustrated in ref. [5].

Quite a number of papers dealing with the issue of perturbative quantization of CST have already appeared [6-10]. This has led to some controversy about the exact meaning of the shift (3) in the perturbative framework. Our main concern here will be to provide a sound perturbative setting for the quantization of CST. A careful analysis of some of the features of the perturbative approach will result in a proper understanding of eq. (3). Our study passes through the determination of the effective action $\Gamma[A]$, the quantum analogue of the classical $S_{\mathrm{CS}}[A]$. The effective action is the generating functional of the 1PI Green functions and therefore the starting point for the computation of any other quantum observable.

We have already mentioned the invariance of $S_{\mathrm{CS}}[A]$ under infinitesimal gauge transformations. Acting with a finite gauge transformation $h, S_{\mathrm{CS}}[A]$ is transformed into [11] (apart from surface terms) to $S_{\mathrm{CS}}\left[{ }^{h} A\right]=S_{\mathrm{CS}}[A]+2 \pi k \mathrm{w}(h)$. The quantity $\mathrm{w}(h)$ is an integer, being the winding number of $h$. In order to have a sigle valued partition function, $k$ must obey the quantization condition $k \in \mathbb{Z}$. Notice that this is a non-perturbative requirement, however, since finite gauge transformations lie beyond the perturbative regime. Furthermore, at the quantum level, the same requirement of monodromy should not be imposed on the classical $k$ but rather on its quantum or renormalized counterpart. We shall come back to this point later on.

To quantize perturbatively CST one must first fix a gauge. We shall work in the Landau gauge, which is known to be free of infrared divergences. With the standard Faddev-Popov construction this amounts to adding to $S_{\mathrm{CS}}[A]$ the term

$$
S_{\mathrm{GF}}=2 \int_{M} d^{3} x \sqrt{g} \operatorname{Tr}\left[\left(J^{\mu}-\partial^{\mu} \bar{c}\right) D_{\mu} c-b \partial_{\mu} A^{\mu}-\frac{1}{2} H[c, c]\right] .
$$

As customary, $b$ denotes the Lagrange multiplier for the gauge condition $\partial_{\mu}\left(\sqrt{g} A^{\mu}\right)=0$, $c$ and $\bar{c}$ are Faddeev-Popov ghosts and $J$ and $H$ are auxiliary fields introduced for later convenience. The relevant aspect of $S_{\mathrm{GF}}$ is that it necessarily picks a metric $g$ on $M$. Thus the resulting gauge-fixed action $S=S_{\mathrm{CS}}+S_{\mathrm{GF}}$ is no longer gauge invariant nor metric independent. The action is, however, BRS invariant. As $S_{\mathrm{GF}}$ is a pure BRS variation, it is not observable. Therefore, at least at the classical level, not only gauge invariance but also the topological character of the theory is recovered. One might then wonder if the same result holds true for the quantized theory.

To carry out our quantization program we choose to work on $M=\mathbb{R}^{3}$ endowed with the flat metric $g_{\mu \nu}=\delta_{\mu \nu}$. Although by naive power counting the theory appears renormalizable, 
it is in fact ultraviolet (UV) finite $[12,13]$, that is, the beta function and the anomalous dimensions of the fields vanish to all orders in perturbation theory. This result should not be very surprising since CST on $S^{3}$, being topological, has no physical local excitations. It was subsequently questioned if UV finiteness of CST implied the vanishing of radiative corrections to the parameter $k$, something which would be in disagreement with the wellestablished non-perturbative result (3). We shall see that this is not going to be the case.

UV perturbative finiteness of Green functions does not imply that the corresponding Feynman diagrams should also be finite. On the contrary, since power counting predicts divergences for individual diagrams, the expected scenario is a cancellation of the divergences order by order in perturbation theory when summing over all diagrams contributing to a given Green function. Although divergences cancel out in the final answer, for practical computational reasons the divergent integrals must be made mathematically manageable by means of a suitable regulator. In this respect CST does not differ from ordinary renormalizable theories.

Let us denote with $\Lambda$ the regulator (or set of regulators) needed to regularize CST at any perturbative order. Since the theory is UV finite, the limit $\Lambda \rightarrow \infty$ in which the regulator is removed defines a "minimal" renormalization scheme where renormalized quantities equal bare ones. In this scheme, that we call renormalized=bare, the renormalized effective action $\Gamma$ and Green functions are defined as

$$
\Gamma=\lim _{\Lambda \rightarrow \infty} \Gamma_{\Lambda}, \quad G\left(p_{1}, \ldots, p_{E}\right)=\lim _{\Lambda \rightarrow \infty} G_{\Lambda}\left(p_{1}, \ldots, p_{E}\right)
$$

where $\Gamma_{\Lambda}$ and $G_{\Lambda}$ are the corresponding regularized quantities. The value of the Green functions so obtained depends in principle on the particular regulatization employed and so does the value of the observables of the theory. However, we shall see that all the known regularization methods satisfying certain invariance requirements lead to observable one-loop radiative corrections that reproduce the non-perturbative results. Thus, these invariance requirements, along with the renormalization scheme above, provide a "natural" parametrization of the perturbative theory.

The BRS symmetry of the gauge fixed action is what is left of the original symmetries of the theory. Classically, the cohomology of the BRS operator guarantees that, when computing observables, the original symmetries still hold true. It is then clear that if we set some hope on obtaining the same picture at the quantum level, we cannot but rely on the BRS symmetry, which is known to be non anomalous in this case $[12,13]$. We then say that BRS is fundamental, meaning with this that it must be enforced at the quantum level in order to make sense out of the quantum theory. It will appear that for CST in the Landau gauge, once BRS symmetry has been implemented on the renormalized theory, the parameter $k$ occurs to be the only actual free parameter. The "natural" parametrization choice is then in terms of the bare (or classical) $k$. This does not specify completely a renormalization scheme because finite renormalizations of the fields are still allowed. However, since the value of the observables is unchanged under finite wave function rescalings, the quantum theory 
is unambigously defined. Actually, we shall make recourse to this freedom of rescaling the fields when comparing Green functions obtained with different regulators. The simplest way to make sure that we get a BRS invariant quantum theory is to start with a BRS invariant regulator so that the effective action we get from (4) in the scheme renormalized=bare satisfies automatically the BRS identity. It will turn out that the observables as functions of the bare $k$ are the same functions of $k+c_{V}$ for all BRS invariant regularization schemes. Obviously, the agreement between perturbative and non-perturbative results in this case originates from the thorough gauge invariance of both quantization methods. It is worth mentioning that with explicitly BRS breaking regularizations, such as the one in ref. [6], the shift (3) is not observed as consequence of the loss of BRS invariance at the regularized level, despite the latter is restored when the regulator is removed.

We then move on to the computation of the most general BRS invariant effective action. It is convenient to introduce the functional

$$
\bar{\Gamma}=\Gamma+2 \int d^{3} x \operatorname{Tr}\left(b \partial_{\mu} A^{\mu}\right)
$$

which, owing to the Landau gauge condition and the antighost equation, is idependent of $b$ and depends on $J_{\mu}$ and $\bar{c}$ only through the combination $G_{\mu}=J_{\mu}-\partial_{\mu} \bar{c}$ [8]. The BRS identity for $\bar{\Gamma}$ takes then the form

$$
\int d^{3} x \operatorname{Tr}\left(\frac{\delta \bar{\Gamma}}{\delta A^{\mu}} \frac{\delta \bar{\Gamma}}{\delta G_{\mu}}+\frac{\delta \bar{\Gamma}}{\delta c} \frac{\delta \bar{\Gamma}}{\delta H}\right)=0
$$

Inserting in eq.(5) the loop expansion $\bar{\Gamma}=\sum_{n=0}^{\infty} \hbar^{n} \bar{\Gamma}_{n}$, we get a tower of equations that must be satisfied order by order in $\hbar$. At first order we have

$$
\Delta \bar{\Gamma}_{1}=0
$$

where $\Delta$ is the Slavonv-Taylor operator

$$
\Delta=\int d^{3} x \operatorname{Tr}\left[\frac{\delta \bar{\Gamma}_{0}}{\delta A^{\mu}} \frac{\delta}{\delta G_{\mu}}+\frac{\delta \bar{\Gamma}_{0}}{\delta G_{\mu}} \frac{\delta}{\delta A^{\mu}}+\frac{\delta \bar{\Gamma}_{0}}{\delta c} \frac{\delta}{\delta H}+\frac{\delta \bar{\Gamma}_{0}}{\delta H} \frac{\delta}{\delta c}\right]
$$

The operator $\Delta$ is nihilpotent and is the quantum generalization of the classical BRS operator.

Eq. (6) is formally identical to the usual stability equation for BRS. However, the cohomological problem to be solved here is by far more difficult, since $\bar{\Gamma}_{1}$ might contain not only local but also non-local contributions. Under the additional hypothesis that contributions involving fewer than four fields be purely local, the local and non-local sectors can be proven to decouple in eq.(6) and we can easily solve for the local part [8]. This locality requirement is justified a posteriory by the explicit computation of the Green functions involving up to 
three external legs. Observe that the latter are the only Green functions that by power counting need to be regularized. Consequently, the local part of $\bar{\Gamma}_{1}$ encodes all the arbitrariness of the regularization scheme used. Of course, terms involving four or more fields are necessarely non-local, if not zero, for dimensional reasons and, being finite, do not depend on the regularization. For the local part of $\bar{\Gamma}_{1}$ we then get [8]

$$
\begin{gathered}
W\left(\alpha_{1}, \beta_{1}, \gamma_{1}\right)=-\frac{i k}{4 \pi} \int d^{3} x \operatorname{Tr}\left[\left(\alpha_{1}+2 \beta_{1}\right) A \wedge \mathrm{d} A+\frac{2}{3}\left(\alpha_{1}+3 \beta_{1}\right) A \wedge A \wedge A\right] \\
-2 \int d^{3} x \operatorname{Tr}\left[\beta_{1} G_{\mu} \partial^{\mu} c-\gamma_{1} G_{\mu}\left(D^{\mu} c\right)+\frac{1}{2} \gamma_{1} H[c, c]\right],
\end{gathered}
$$

where $\alpha_{1}, \beta_{1}$ and $\gamma_{1}$ are regularization dependent (finite) coefficients. They correspond to the freedom of renormalizing $k$ and the fields. The (local part of the) one loop effective action can then be recast in the form

$$
\Gamma^{\mathrm{loc}}=\left(1+\alpha_{1}\right) S_{C S}[A]-2 \int d^{3} x \operatorname{Tr}\left(b \partial_{\mu} A^{\mu}\right)+\Delta X\left(\beta_{1}, \gamma_{1}\right) .
$$

Here $X\left(\beta_{1}, \gamma_{1}\right)=4 \int d^{3} x \operatorname{Tr}\left[\beta_{1} G^{\mu} A_{\mu}-\left(1+\gamma_{1}\right) H c\right]$. The effective action receives two different kinds of contributions. One (corresponding to $\alpha_{1}$ ) is gauge invariant, metric independent and provides a monodromy parameter $\left(1+\alpha_{1}\right) k$. The other is the term $\Delta X$ which, being cohomologically trivial, does not contribute to to the expectation values of Wilson loops [10]. These properties can be made manifest by the wave function renormalization $\Phi_{R}=Z_{\Phi} \Phi$ $(\Phi=A, b, c, H, G)$, with $Z_{A}=Z_{G}^{-1}=Z_{b}^{-1}=1+\beta_{1}, Z_{c}=Z_{H}^{-1}=1+\gamma_{1}$, so that $\Gamma^{\text {loc }}$ takes the form

$$
\Gamma^{\mathrm{loc}}=\left(1+\alpha_{1}\right) S_{\mathrm{CS}}\left[A_{R}\right]+S_{\mathrm{GF}}\left[\Phi_{R}\right]
$$

Since $\alpha_{1}$, the only observable one-loop correction, could still depend on the regularization, one would conclude that the emerging quantum theory is not unique. As anticipated, however, all BRS invariant regulators used so far for CST yield the same value for $\alpha_{1}$. In the folowing table we collect the values (in units of $c_{V} / k$ ) of the one-loop parameters for the following BRS invariant regularization schemes: large mass limit of dimensionally regularized topologically massive Yang-Mills (TMYM) theory [8], $\eta$-function regularization [2], higher covariant derivatives (HCD) and Pauli-Villars fields $[7,10]$ and geometric regularization [9].

\begin{tabular}{|c|c|c|c|}
\hline Regularization Method & $\alpha_{1}$ & $\beta_{1}$ & $\gamma_{1}$ \\
\hline Large $m$ of TMYM + dimensional reg. & 1 & $2 / 3$ & 0 \\
\hline$\eta$-function regularization & 1 & 0 & 0 \\
\hline HCD + Pauli-Villars & 1 & $2 / 9$ & 0 \\
\hline Geometric regularization & 1 & $4 /(3 \pi) I_{n}$ & $*$ \\
\hline
\end{tabular}

Geometric quantization makes use of ghost generations different from the standard Fad-

* The values given here for HCD+Pauli-Villars are those computed in [10] rather than those in [7], where strictly speaking only Pauli-Villars fields and no HCD are used. In [7] one gets $\alpha_{1}=1, \beta_{1}=\gamma_{1}=0$. 
deev-Popov ones, so only the pure gauge sector of $\Gamma^{\text {loc }}$ can be compared; the quantity $I_{n}$ is defined as $I_{n}=\int_{0}^{\infty} d p \frac{\left(1+p^{2}\right)^{n}}{1+p^{2}\left(1+p^{2}\right)^{2 n}}$, with $n>1$ an arbitrary integer. As it appears evident, different regularizations yield different $\Gamma$ 's but all of them predict the same observable shift $k \rightarrow k+c_{V}$ and therefore the same physical theory. This is in agreement with the nonperturbative result (3) and leads to a self-consistent, single valued quantum theory, since the monodromy parameter $k+c_{V}$ keeps beeing an integer.

At this point the obvious question arises: what does it happen at higher perturbative orders? Now that we obtained the solution (7) for the one loop effective action, we can proceed and solve the BRS identity at order $\hbar^{2}$. If $\bar{\Gamma}_{2}$ has the same locality properties as $\bar{\Gamma}_{1}$, its local part is found to be $[8]$

$$
\bar{\Gamma}_{2}^{\mathrm{loc}}=W\left(\alpha_{2}, \beta_{2}, \gamma_{2}\right)+\frac{i k}{4 \pi} \int d^{3} x \beta_{1}\left(\alpha_{1}+3 \beta_{1}-\gamma_{1}\right) \operatorname{Tr}(A \wedge \mathrm{d} A)
$$

so that the (local part of) the two-loop effective action reads

$$
\Gamma^{\mathrm{loc}}=\Gamma_{0}+W\left(\alpha_{1}+\alpha_{2}, \beta_{1}+\beta_{2}, \gamma_{1}+\gamma_{2}\right)+\frac{i k}{4 \pi} \int d^{3} x \beta_{1}\left(\alpha_{1}+3 \beta_{1}-\gamma_{1}\right) \operatorname{Tr}(A \wedge \mathrm{d} A)
$$

The three new parameters $\alpha_{2}, \beta_{2}$ and $\gamma_{2}$ are second order and regularization scheme dependent. The apparently awkward expression (9) takes a simple and familiar form if we subtract the one loop contributions corresponding to $\Delta X\left(\beta_{1}, \gamma_{1}\right)$ entering the two-loop diagrams through one loop subdiagrams. This is accomplished by means of the same wave function renormalization leading to eq. (7). In terms of the renormalized fields we have

$$
\Gamma_{\text {local }}=\left(1+\alpha_{1}+\tilde{\alpha}_{2}\right) S_{C S}\left[A_{R}\right]-2 \int d^{3} x \operatorname{Tr}\left(b_{R} \partial_{\mu} A_{R}^{\mu}\right)+\Delta_{R} X_{R}\left(\tilde{\beta}_{2}, \gamma_{2}\right)
$$

where the subsript $R$ indicates renormalized quantities and $\tilde{\alpha}_{2}=\alpha_{2}-6\left(\beta_{1}\right)^{2}-3 \alpha_{1} \beta_{1}+3 \beta_{1} \gamma_{1}$, $\tilde{\beta}_{2}=\beta_{2}+\beta_{1}^{2}-\beta_{1} \gamma_{1}$. Just like at one loop, BRS invariance implies that out of three parameters, only one, $\tilde{\alpha}_{2}$, is gauge invariant hence observable as a shift of $k$. Thus, from a perturbative viewpoint a two-loop correction to $k$ would be allowed. Note that now $\tilde{\alpha}_{2}$ is order $\left(c_{V} / k\right)^{2}$ and cannot possibly lead to an integer shift for any integer choice of $k$. Finding a non-zero $\tilde{\alpha}_{2}$ would imply an incompatibility between the non-perturbative request of single valuedness and perturbative quantization. The computation of $\tilde{\alpha}_{2}$ in a BRS invariant regularization scheme is then particularly intriguing. In ref. [8] we showed how to compute the two-loop effective action for the first BRS invariant regularization scheme listed in the table above. In the remaining part of this note we shall briefy summarize the ideas and the results of the method, warmly inviting the interested reader to refer back to the original paper where more details can be found.

Dimensional regularization ensures BRS invariance and algebraic consistency if we start with a $D$ dimensional extended action $S_{\mathrm{CS}}[A]$ with the following prescription for the $\epsilon_{\mu \nu \rho}$ 
symbol (showing up once $S_{\mathrm{CS}}[A]$ is written in coordinates). The $D$-dimensional analogue of $\epsilon_{\mu \nu \rho}$ is defined as a completely antisymmetric object in its indices satisfying the following identities [14]:

$$
\epsilon_{\mu_{1} \mu_{2} \mu_{3}} \epsilon_{\nu_{1} \nu_{2} \nu_{3}}=\sum_{\pi \in \mathcal{S}_{3}} \operatorname{sign}(\pi) \prod_{i=1}^{3} \tilde{g}_{\mu_{i} \nu_{\pi(i)}} \quad, \quad \epsilon_{\mu_{1} \mu_{2} \mu_{3}} \hat{g}^{\mu_{3} \mu_{4}}=0 .
$$

Here $g_{\mu \nu}=\tilde{g}_{\mu \nu} \oplus \hat{g}_{\mu \nu}$ is the euclidean metric in $D$ dimensions, with $\tilde{g}_{\mu \nu}$ and $\hat{g}_{\mu \nu}$ its 3 - and $(D-3)$-dimensional projections respectively. Furthermore, given a $D$-dimensional vector $u^{\mu}$ we define $\hat{u}^{\mu}=\hat{g}^{\mu \nu} u_{\nu}$ and $\tilde{u}^{\mu}=\tilde{g}^{\mu \nu} u_{\nu}$. Notice that objects with a hat vanish when $D \rightarrow 3$; they are called evanescent.

Unfortunately, pure CST theory with the above prescription for the $\epsilon_{\mu \rho \nu}$ symbol, has a non-invertible kinetic term, even in a general $\alpha$-gauge, because of the zero modes $z(p)=$ $f(p)\left(\hat{p}^{2} \hat{g}_{\mu \nu}-\hat{p}_{\mu} \hat{p}_{\nu}\right)$. This hinders a perturbative analysis. To get out of this empasse we can add to $S_{\mathrm{CS}}[A]$ a Yang-Mills term

$$
S_{\mathrm{YM}}[A]=\frac{k}{8 \pi m} \int d^{3} x \operatorname{Tr}\left(F_{\mu \nu} F^{\mu \nu}\right),
$$

where $F_{\mu \nu}$ is the field strength of the gauge field $A_{\mu}$. The theory defined by the action $S_{m}[A]=S_{\mathrm{CS}}[A]+S_{\mathrm{YM}}[A]$ corresponds to so the called Topologically Massive Yang Mills theory (TMYM), proposed in ref. [11] to provide a way (alternative to the usual Higg mechanism) for making the gauge field massive without losing gauge invariance. The parameter $m$ is the bare topological mass of the gauge field and CST is recovered in the limit of infinite mass gap. The TMYM theory, differently from CST, is superrenormalizable and only oneand two-loop diagrams are divergent. Therefore, from our viewpoint, $m$ can be envisioned as an UV regulator. The regularized CST is then defined in the limits $m \rightarrow \infty$ and $D \rightarrow 3$. These two limits do not commute and, to have a consistent BRS invariant regularization prescription, one must take $D \rightarrow 3$ first. Only in the case that the limit $D \rightarrow 3$ does not give rise to divergences, is one allowed to let $m \rightarrow \infty$. Therefore, if our regularization prescription has to make sense, TMYM theory must be UV finite. That this is indeed the case has been shown in ref. [8].

The addition of a Yang-Mills term to the Chern-Simons action entails several side effects. From a non-perturbative, functional point of view, the wild oscillatory behaviour of the path integral (2) is tamed by the presence of the Yang-Mills contribution. Thus the TMYM path integral is in fact a regularized version of Chern-Simons one. The price paied for this regulating effect is a total change in the structure of the Hilbert space. Indeed TMYM theory is not topological and the gauge fields excitations are propagating. In the limit of infinite mass gap the propagating modes decouple from the non-propagating ones, and CST is reobtained. What we are left with is thus the (degenerate) ground state of TMYT which precisely corresponds to the zero-energy Hilbert space of CST [15]. This picture is reminiscent of Klauder's regularization prescription of the path integral for quantum mechanical systems, which is known to be completely equivalent to geometric quantization [16]. 
The definition (10) for the $D$-dimensional $\epsilon_{\mu \nu \rho}$ makes the formal theory invariant under $S O(3) \otimes S O(D-3)$, rather than $S O(D)$. As a result, the Feynman rules involve hatted and twiddled objects in a non-trivial way. For the gauge propagator we have (dropping colour indices): $D_{\mu \nu}(\tilde{p}, \hat{p})=\Delta_{\mu \nu}(p)+R_{\mu \nu}(\tilde{p}, \hat{p})$, where $\Delta_{\mu \nu}(p)$ and $R_{\mu \nu}(\tilde{p}, \hat{p})$ are given by

$$
\begin{gathered}
\Delta_{\mu \nu}(p)=\frac{4 \pi}{k} \frac{m}{p^{2}\left(p^{2}+m^{2}\right)}\left(m \epsilon_{\mu \rho \nu} p^{\rho}+p^{2} g_{\mu \nu}-p_{\mu} p_{\nu}\right) \\
R_{\mu \nu}(\tilde{p}, \hat{p})=\frac{4 \pi}{k} \frac{m^{3}}{p^{2}\left[\left(p^{2}\right)^{2}+m^{2} \tilde{p}^{2}\right]}\left[\frac{\hat{p}^{2}}{p^{2}+m^{2}}\left(\epsilon_{\mu \rho \nu} p^{\rho}+p^{2} g_{\mu \nu}+\frac{m^{2}}{p^{2}} p_{\mu} p_{\nu}\right)\right. \\
\left.+\tilde{p}^{2} \hat{g}_{\mu \nu}+\hat{p}_{\mu} \hat{p}_{\nu}-p_{\mu} \hat{p}_{\nu}-\hat{p}_{\mu} p_{\nu}\right] .
\end{gathered}
$$

Notice that $R_{\mu \nu}(\tilde{p}, \hat{p})$ is vanishing in the limit $D \rightarrow 3$. It is obvious that vanishing objects do not contribute at the tree level. At higher perturbative orders however, they could combine with divergent terms and yield finite contributions. A careful study [8] of the convergence properties of $1 \mathrm{PI}$ Green functions having at least one $R_{\mu \nu}(\tilde{p}, \hat{p})$ insertion leads to the conclusion that they are finite and hence vanishing at $D=3$. Therefore we can use $\Delta_{\mu \nu}(p)$ as the "effective" free gauge field propagator.

The determination of $\bar{\Gamma}_{2}^{\text {loc }}$ necessitates of the knowledge of three independent Green functions. It is wise to choose the simplest ones, namely the vacuum polarization, the ghost self-energy and the $H c c$ vertex. The explicit computation of these Green functions presents, from the point of view of the algebra involved, the same degree of complexity as QCD. The situation is much worse from the point of view of integration, since now we are faced with massive denominators. The key observation that makes the computation feasible is that we are only interested in the asymptotic behaviour of the integrals for large values of $m$. We will then use two theorems theorems that enable us to tell if an integral is vanishing in the limit $m \rightarrow \infty$ or not.

A general integral from a $L$-loop Feynman diagram is of the form

$$
I(p, m)=m^{\beta} \int d k \frac{M(k)}{\prod_{i}\left(l_{i}^{2}\right)^{n_{i}} \prod_{j}\left(l_{j}^{2}+m^{2}\right)^{n_{j}}},
$$

where the integration measure is $d k=d^{3} k_{1} \cdots d^{3} k_{L}, \beta$ denotes an arbitrary real number and $n_{i}$ are positive integers. The momenta $l_{i}$ are linear combinations of internal and external momenta. The numerator $M(k)$ is a monomial of degree $n_{k}$ in the components of the vectors $k_{1}, \ldots, k_{L}$. We call $d$ to the mass dimension of $I(p, m)$ and denote by $\underline{\omega}_{\text {min }}$ the minimum between zero and the lowest infrared degree at zero external momenta of all the subintegrals of $I(p, m)$, including $I(p, m)$ itself. Then the following theorem holds: 
Theorem 1. If the integral $I(p, m)$ is both $U V$ and IR convergent by power counting at non-exceptional external momenta, and the mass dimension $d$ and $\underline{\omega}_{\min }$ defined above satisfy $d-\underline{\omega}_{\min }<0$, then $I(p, m)$ vanishes when $m$ goes to $\infty$.

To formulate the second vanishing theorem we introduce the notation $[n]=0$ for $n$ even and $[n]=1$ for $n$ odd. The theorem states then:

Theorem 2. If the integral $I(p, m)$ in (11) is absolutely convergent at zero external momenta and its mass dimension $d$ satisfies $\left[n_{k}\right]>d$, then $I(p, m) \rightarrow 0$ as $m \rightarrow \infty$.

Concerning the hypothesis of UV convergence in these two theorems, we must mention that it can in fact be relaxed [8] for the case of TMYM, at least up to two loops, to the much weaker condition that the integral (11) be finite in dimensional regularization.

In the limits $D \rightarrow 3$ and $m \rightarrow \infty$, the result we obtained for the second order coefficients appearing in the two-loop effective action (9) are the following

$$
\alpha_{2}=\frac{14}{3}\left(\frac{c_{V}}{k}\right)^{2} \quad, \quad \beta_{2}=\frac{169+L}{72}\left(\frac{c_{V}}{k}\right)^{2}, \quad \gamma_{2}=0
$$

with $L=528 \ln 2-567 \ln 3$. Plugging these values into the expression of $\tilde{\alpha}_{2}$ and taking into account the values of the one-loop parameters in the table, we conclude that $\tilde{\alpha}_{2}$ is zero and therefore the two-loop corrections do not have observable consequences. Stated differently, there is no two-loop shift of the parameter $k$ owing to the cohomologically trivial character of the second order corrections. We conjecture that this picture holds true also at higher perturbative corrections, despite an explicit calculation is still lacking.

It would be also nice to have at our disposal the corresponding two-loop results for the other BRS invariant regularizations listed in the previous table, so to get a thorough check of the uniqueness of the quantum theory parameterized in terms of the bare $k$. Unfortunately, what we have presented here is, at present, the only instance where a two-loop computation has beed carried out for CST with a manifestely BRS invariant regularization scheme. 


\section{REFERENCES}

1. For a recent review see for example:

R. Iengo and K. Lechner, Phys. Rep. 213 No. 4 (1992) 179.

2. E. Witten, Commun. Math. Phys. 121 (1989) 351.

3. V.F.R. Jones, Ann. Math. 126 (1987) 335.

4. D.S. Freed and R.E. Gompf, Phys Rev. Lett. 66 (1991) 1255.

5. E. Guadagnini, M. Martellini and M. Mintchev, Nucl. Phys. B330 (1990) 575.

6. E. Guadagnini, M. Martellini and M. Mintchev, Phys. Lett. 224 (1989) 489.

7. L. Alvarez-Gaumé, J.M.F. Labastida and A.V. Ramallo, Nucl. Phys. B334 (1990) 103.

8. G. Giavarini, C.P. Martin and F. Ruiz Ruiz, Nucl. Phys. B381 (1992) 222.

9. M. Asorey and F. Falceto, Phys. Lett. 241 (1990) 31; Int. J. Mod. Phys. A7 No.2 (1992) 235.

10. G. Giavarini, F. Ruiz Ruiz, C.P. Martin, Perturbative quantization of Chern-Simons theory, preprint.

11. S. Deser, R. Jackiw and S. Templeton, Ann. Phys. 140 (1982) 372.

12. A. Blasi and R. Collina, Nucl. Phys. B345 (1990) 472.

13. F. Delduc, C, Lucchesi, O. Piguet and S.P. Sorella, Nucl. Phys. B346 (1990) 513.

14. P. Breitenlohner and D. Maison, Commun. Math. Phys. 52 (1977) 11.

15. A.P. Polychronakos, Ann. of Phys. 203, No.2, (1990) 231.

16. J. Klauder and E. Onofri, Int. J. Mod. Phys. A4 (1989) 15. 